\section{Case Reports in Oncology}

Case Rep Oncol 2016;9:298-304

DOI: $10.1159 / 000445827$

Publisned oniıne: June 11, 2016
(C) 2016 The Author(s)

Published by S. Karger AG, Basel

$1662-6575 / 16 / 0092-0298 \$ 39.50 / 0$

www.karger.com/cro

This article is licensed under the Creative Commons Attribution-NonCommercial 4.0 International License (CC BY-NC) (http://www.karger.com/Services/OpenAccessLicense). Usage and distribution for commercial purposes requires written permission.

\title{
Human Epidermal Growth Factor Receptor 2 (HER-2/neu)-Directed Therapy for Rare Metastatic Epithelial Tumors with HER-2 Amplification
}

\author{
Daniel Sanghoon Shin ${ }^{a, b}$ Timothy Sherry $^{c}$ Michael E. Kallen $^{d}$ \\ Steven Wong ${ }^{a}$ Alexandra Drakaki ${ }^{\mathrm{a}}$ \\ ${ }^{a}$ Division of Hematology-Oncology, Department of Medicine, and Departments of \\ ${ }^{b}$ Molecular, Cellular and Integrative Physiology, ${ }^{C}$ Molecular, Cellular and Developmental \\ Biology and ${ }^{d}$ Pathology and Laboratory Medicine, University of California Los Angeles, \\ Los Angeles, Calif., USA
}

\section{Key Words}

Human epidermal growth factor receptor 2 - Salivary duct carcinoma - Extramammary Paget's disease of the scrotum

\begin{abstract}
Case 1: A 67-year-old Asian female was diagnosed with locally advanced high-grade salivary duct carcinoma in June 2011. Molecular analysis revealed human epidermal growth factor receptor 2 (HER-2) amplification. She received adjuvant therapy with carboplatin/paclitaxel/ trastuzumab and maintenance of trastuzumab. Upon disease progression, trastuzumab could not be continued due to lack of financial coverage. Instead, she was treated with compassionate use of lapatinib from April 2013 and standard 5-fluorouracil. Her disease ultimately progressed and she expired later in 2013. Case 2: A 68-year-old Asian male was diagnosed with extramammary Paget's disease of the scrotum with HER-2 amplification in May 2011. He received 6 cycles of adjuvant trastuzumab/docetaxel/carboplatin followed by maintenance trastuzumab, which was changed to compassionate use of lapatinib as his insurance did not cover further administration of trastuzumab. He showed clinical benefits from single-agent lapatinib and a combination of lapatinib/capecitabine upon progression to the single-agent lapatinib. Ultimately, he was started on ado-trastuzumab emtansine, which was approved at that time by the FDA for HER-2-positive breast cancer progressed on trastuzumab. He is
\end{abstract}

\section{KARGER}

Daniel Sanghoon Shin, MD

Division of Hematology-Oncology

University of California Los Angeles

10945 Le Conte Ave, Suite 2333A, Los Angeles, CA 90095 (USA)

E-Mail dsshin@mednet.ucla.edu 
Shin et al.: Human Epidermal Growth Factor Receptor 2 (HER-2/neu)-Directed Therapy for Rare Metastatic Epithelial Tumors with HER-2 Amplification

having clinical and radiographic complete response based on current imaging and normalization of his tumor markers. Conclusion: HER-2-targeted therapy should be considered for tumors with HER-2 amplification. In our case series, we would like to emphasize this approach in other rare histologies. Specifically, our patient with extramammary Paget's disease of the scrotum represents the first reported case of a non-breast, non-gastric tumor with HER-2 overexpression with complete clinical and radiographic response to HER-2-targeted therapy.

(C) 2016 The Author(s)

Published by S. Karger AG, Basel

\section{Background}

The discovery of the role of human epidermal growth factor receptor 2 (HER-2) in breast cancer research was the result of the observation of amplified HER-2 in breast cancer cell line models, prompting analysis of clinical breast cancer specimens. This translational breast cancer research effort was driven by its prevalence, public awareness and high level of interest leading to significant sources of funding and research focus in pursuing development of effective therapies. The initial finding, published in 1987 [1], then identified a subset of breast cancer with the worst prognosis when compared with hormone-positive disease. In 1998, the FDA approval of trastuzumab for metastatic HER-2-positive breast cancer and subsequent studies in earlier-stage disease as well as the development of further HER-2 inhibitors [lapatinib, pertuzumab, ado-trastuzumab emtansine (T-DM1)] have now placed HER-2-positive breast cancer among those diseases with remarkably more favorable prognoses compared to hormone receptor-positive diseases. This experience as well as this case series of identifying HER-2 amplifications in rare tumors highlight the importance of the HER-2 amplification pathway, and how drugs like trastuzumab can be utilized in these different cancers with the HER-2 amplification.

While one would expect that those treatments will be important for treatment of tumors overexpressing HER-2, based on the current oncology guidelines (NCCN, ASCO) this is not the standard of care and up until recently most FDA-approved therapies are based on histology. With the novel approach in melanoma research, the scientific community is changing the clinical practice; however, most tumors are still being treated based on the underlying pathology. The problem with the approach of relying on histologic primary disease to define which patient population to study, is that it will inherently neglect uncommon or rare tumors where accrual of adequate numbers of patients for these clinical trials would be next to impossible.

Comprehensive molecular screening of tumors and target identification has been studied by Von Hoff et al. [2] utilizing the Caris platform with positive results supporting further study of this approach. Given this other groups/commercial diagnostics (theranostics), companies have emerging technologies to further support this effort (Foundation One Medicine, Guardant 360, Biotheranostics, Inc., Cellnetix, etc.). However, despite the ability to screen tumors for a predictive biomarker such as amplified HER-2, to date, registrational studies based on molecular tumor data without respect to primary tumor type have been lacking; thus, FDA approval and insurance coverage for these patients is not routinely supported.

The purpose of this report is to illustrate two cases of relatively uncommon or rare diseases where a molecular screen surprisingly revealed a HER-2 amplification suggesting consideration for HER-2-directed therapy. Those HER-2-positive cases are salivary duct carcinoma (SDC) and extramammary Paget's disease (EMPD) of the scrotum. 
Shin et al.: Human Epidermal Growth Factor Receptor 2 (HER-2/neu)-Directed Therapy for Rare Metastatic Epithelial Tumors with HER-2 Amplification

SDCs are highly aggressive tumors with poor 5-year survival and $20-25 \%$ of patients present with metastatic disease [3]. Molecular oncogenesis in salivary gland tumor has been reported in a number of studies [4] and the HER-2 expression and the association with clinical outcome has been controversial [3]. Combination of taxanes and trastuzumab is associated with some responses. A phase II trastuzumab monotherapy trial [5] in metastatic salivary gland tumor was closed early due to the low rate of positivity of HER-2 overexpression; however, one patient received 40 cycles of trastuzumab with partial response.

EMPD is a rare malignant skin tumor and mainly occurs on skin appendages, regions rich in apocrine glands such as scrotum, vulva and axilla. Approximately, $20 \%$ of patients have metastatic disease at presentation, which carries poor prognosis. Currently, there are essentially no reliably effective treatments available for advanced cases [6]. HER-2-positive EMPD is extremely rare with only a few case reports. It has been suggested that HER-2 receptor contributes to the intra-epidermal spread of Paget cells, cellular proliferation as well as migration [7]. Tanaka et al. [8] reported over 100 cases of EMPD, and found approximately $15 \%$ of them had HER-2 overexpression which correlated with biologically aggressive disease with a few cases of HER-2-positive EMPD, treated with trastuzumab with clinical benefit. However, this is the first reported case of a patient with metastatic EMPD of the scrotum who has complete response to HER-2-directed therapy with T-DM1.

\section{Case Presentation}

\section{Case 1: Salivary Duct Carcinoma}

A 67-year-old Asian female presented with an enlarging mass near her left parotid gland in June 2011 which had grown until she sought medical attention 3 months after initially noticing the mass. She did not have any significant comorbidities. MRI in September 2011 showed an ill-defined, deep $3.7 \times 3 \times 3 \mathrm{~cm}$ left parotid gland and enlarged left cervical lymph nodes measuring up to $2.2 \mathrm{~cm}$. Following PET-CT showed numerous hypermetabolic nodes in the ipsilateral neck levels II-V; however, there was no clear evidence of metastasis. She underwent biopsy of the left parotid mass as well as lymph nodes, which revealed epithelial neoplasm of uncertain origin. She was evaluated by head and neck surgery at UCLA and was noted to have a firm 5-cm left parotid mass as well as paralysis of the left marginal mandibular nerve and involuntary spasm of the left orbicularis oris and orbicularis oculi muscles. The patient underwent left total parotidectomy (sacrificing facial nerve) and modified neck dissection from levels I-V with neurolysis and preservation of the spinal accessory nerve.

\section{Laboratory and Diagnostic Summary}

The pathology showed high-grade SDC, a 6.1-cm parotid mass in its greatest dimension, extending into extraparotid soft tissue with extensive perineural/intraneural invasion as well as lymphatic and vascular invasion. The surgical margins were negative. Two of two intraparotid lymph nodes were positive for metastatic SDC. Thirty-one of sixty-seven lymph nodes were positive for metastatic high-grade SDC with extranodal extension. The vertical segment of her facial nerve was involved with clear surgical margins. Immunohistochemistry was positive for CK7, AR, mammaglobin, GCDFP15, and HER-2 with 3+. CK5/6 was focally positive and CK20 was negative. Subsequent FISH for HER-2 gene amplification was positive (fig. 1; R/G ratio, the ratio of HER-2 signal to centromere 17 signal, was 8.7. Ratio defined as 0.6 to 1.6 is negative, greater than 2.2 is positive and between 1.6 and 2.2 is equivocal). 
Shin et al.: Human Epidermal Growth Factor Receptor 2 (HER-2/neu)-Directed Therapy for Rare Metastatic Epithelial Tumors with HER-2 Amplification

Management and Outcome

She received adjuvant chemoradiation with carboplatin/paclitaxel/trastuzumab followed by trastuzumab every 3 weeks from June 2012. Capecitabine was added in September 2012 due to cutaneous disease that represented dermal metastasis on the neck. Repeat biopsy of the lesion confirmed high-grade carcinoma and HER-2 testing showed increased R/G ratio to 13.0 compared to 8.7 from initial biopsy. She experienced hand-foot syndrome from capecitabine, which required a dose adjustment. She had initial improvement in erythema and pain of the cutaneous lesion but eventually progressed. Given this worsening cutaneous disease, her regimen switched to FOLFOX without response. Subsequently, she received concomitant radiation treatment, followed by HER-2-directed therapy with trastuzumab and lapatinib, an oral tyrosine kinase inhibitor that interrupts HER-2 and epidermal growth factor receptor pathways. Unfortunately, trastuzumab administration was not continued due to lack of financial coverage from April 2013. Her disease eventually progressed and she expired in late 2013.

\section{Case 2: Extramammary Paget's Disease of the Scrotum}

A 68-year-old Asian male was referred to UCLA for chronic skin condition on his penile, scrotal area, for which he received multiple antibiotic treatments. Eventually, he underwent a skin biopsy by a dermatologist that demonstrated EMPD of the scrotum.

Laboratory and Diagnostic Summary. The patient underwent surgical excision on March 2011. Staging CT scan did not reveal any adenopathy, although it showed 2 pulmonary micronodules. Fig. 2 shows the pathologic confirmation of HER-2 amplification.

Management and Outcome. The patient presented a year later with local recurrence manifested as inguinal adenopathy. He underwent CT-guided biopsy that showed adenocarcinoma, which was consistent with EMPD originating from the scrotum. Additional molecular study revealed HER-2 positivity via FISH testing and the CT scan was negative for distant metastatic disease. He then underwent left radical superficial and deep inguinal node dissection as well as bilateral pelvic node dissection in July 2012. He received 6 cycles of adjuvant trastuzumab, docetaxel, and carboplatin completed by December 2012 followed by maintenance trastuzumab. His insurance stopped covering trastuzumab and therefore, treatment was discontinued. During that time, his disease appeared to be progressing based on increasing retroperitoneal lymphadenopathy. He underwent repeat biopsy of the lymph nodes confirming EMPD. Interestingly, his malignancy responded to reintroduction of HER-2targeted therapy with lapatinib. He remained on this single-agent treatment for several months; however, his course was complicated by liver function test abnormalities. Capecitabine was added due to inability to maintain full dosing of lapatinib as well as progressive disease in restaging PET-CT. Upon progression, he was started on T-DM1. At the time of this report, he has been in active treatment for almost a year and he continues to have radiologic complete response.

\section{Discussion}

It was a breakthrough discovery by Dr. Dennis Slamon's research group in the 1980s that HER-2 gene amplification/overexpression was linked to more aggressive breast cancer. The identification of the HER-2 pathway led to the era of 'targeted therapy' in cancer management [1]. Trastuzumab was approved in 1998 based on unprecedented clinical activity among $20-25 \%$ of a subset of breast cancer patients who had HER-2 amplification/overexpression [9]. HER-2-directed therapy had been mainly used for breast cancer 
Shin et al.: Human Epidermal Growth Factor Receptor 2 (HER-2/neu)-Directed Therapy for Rare Metastatic Epithelial Tumors with HER-2 Amplification

patients up until the TOGA trial showed the clinical activity on $10-15 \%$ of patients with metastatic gastric cancer that have HER-2 amplification/overexpression [10]. HER-2 amplification/overexpression on other epithelial malignancies such as bladder, colon and lung has been identified, although the clinical activity of trastuzumab is not as impressive as shown with breast cancer [11]. Recent reports suggested that HER-2 expression is not associated with colon cancer prognosis [12]; however, HER-2-targeted therapeutic application for these cancers are still ongoing, especially with dual targeted strategies for colon cancer as we learned from breast cancer studies [13]. In this case series, we would like to highlight the importance of identification of HER-2 amplification/overexpression in uncommon types of epithelial malignancies with the development of molecular profiling as well as next-generation sequencing techniques [14].

In this case series, we reported two rare HER-2-amplified epithelial malignancies: salivary ductal adenocarcinoma, EMPD, and HPV-positive oral cavity squamous cell carcinoma. Given the rarity or scarcity of these cases, it is less feasible to conduct clinical trials based on primary histology to show the potential benefit of HER-2-directed therapy, which is required for FDA approval. Although those two patients were recommended for HER-2-directed therapy, their course of treatment was interrupted by lack of insurance coverage. It may be worth considering a discussion in another context about the possible ethical issues raised by the limitation of their access to the best available therapy.

\section{Conclusion}

The HER-2 signaling pathway is ubiquitously expressed in epithelia and aberrant expression increasingly identified in a subset of many epithelial cancers, certainly not unique to breast or gastric tumors. The experience that was obtained from the trastuzumab use in breast cancer suggests that treatment should be based on the biomarker status (with the main example being the HER-2 status). It is very important for the medical and scientific community but most importantly for the third party payers to recognize that those rare tumors could still be managed with already FDA-approved drugs for other indications. The field should evolve and treatment should also consider the molecular background of those tumors rather than the organ that the tumor is originating from.

\section{Statement of Ethics}

Written informed consents were obtained from the patients for publication of this case series and any accompanying images. The copies of the written consents are available for review by the Editor-in-Chief of this journal.

\section{Disclosure Statement}

The authors declare no competing interests. 


\section{Case Reports in Oncology}

\begin{tabular}{l|l}
\hline Case Rep Oncol 2016;9:298-304 \\
\hline DOI: $10.1159 / 000445827$ & $\begin{array}{l}\text { (c) 2016 The Author(s). Published by S. Karger AG, Basel } \\
\text { www.karger.com/cro }\end{array}$ \\
\hline
\end{tabular}

Shin et al.: Human Epidermal Growth Factor Receptor 2 (HER-2/neu)-Directed Therapy for Rare Metastatic Epithelial Tumors with HER-2 Amplification

\section{References}

1 Slamon DJ, Godolphin W, Jones LA, Holt JA, Wong SG, Keith DE, et al: Studies of the HER-2/neu protooncogene in human breast and ovarian cancer. Science 1989;244:707-712.

-2 Von Hoff DD, Stephenson JJ Jr, Rosen P, Loesch DM, Borad MJ, Anthony S, et al: Pilot study using molecular profiling of patients' tumors to find potential targets and select treatments for their refractory cancers. J Clin Oncol 2010;28:4877-4883.

-3 Jaehne M, Roeser K, Jaekel T, Schepers JD, Albert N, Loning T: Clinical and immunohistologic typing of salivary duct carcinoma: a report of 50 cases. Cancer 2005;103:2526-2533.

-4 Williams MD, Roberts D, Blumenschein GR Jr, Temam S, Kies MS, Rosenthal DI, et al: Differential expression of hormonal and growth factor receptors in salivary duct carcinomas: biologic significance and potential role in therapeutic stratification of patients. Am J Surg Pathol 2007;31:1645-1652.

5 Haddad R, Colevas AD, Krane JF, Cooper D, Glisson B, Amrein PC, et al: Herceptin in patients with advanced or metastatic salivary gland carcinomas. A phase II study. Oral Oncol 2003;39:724-727.

-6 Pascual JC, Perez-Ramos M, Devesa JP, Kutzner H, Requena L: Extramammary Paget's disease of the groin with underlying carcinoma and fatal outcome. Clin Exp Dermatol 2008;33:595-598.

7 Reich 0, Liegl B, Tamussino K, Regauer S: p185HER2 overexpression and HER2 oncogene amplification in recurrent vulvar Paget's disease. Mod Pathol 2005;18:354-357.

-8 Tanaka R, Sasajima Y, Tsuda H, Namikawa K, Tsutsumida A, Otsuka F, et al: Human epidermal growth factor receptor 2 protein overexpression and gene amplification in extramammary Paget disease. Br J Dermatol 2013;168:1259-1266.

-9 Pegram MD, Lipton A, Hayes DF, Weber BL, Baselga JM, Tripathy D, et al: Phase II study of receptorenhanced chemosensitivity using recombinant humanized anti-p185HER2/neu monoclonal antibody plus cisplatin in patients with HER2/neu-overexpressing metastatic breast cancer refractory to chemotherapy treatment. J Clin Oncol 1998;16:2659-2671.

10 Bang YJ, Van CE, Feyereislova A, Chung HC, Shen L, Sawaki A, et al: Trastuzumab in combination with chemotherapy versus chemotherapy alone for treatment of HER2-positive advanced gastric or gastrooesophageal junction cancer (ToGA): a phase 3, open-label, randomised controlled trial. Lancet 2010;376:687-697.

11 Gatzemeier U, Groth G, Butts C, Van ZN, Shepherd F, Ardizzoni A, et al: Randomized phase II trial of gemcitabine-cisplatin with or without trastuzumab in HER2-positive non-small-cell lung cancer. Ann Oncol 2004;15:19-27.

-12 Seo AN, Kwak Y, Kim DW, Kang SB, Choe G, Kim WH, et al: HER2 status in colorectal cancer: its clinical significance and the relationship between HER2 gene amplification and expression. PLoS One 2014;9:e98528.

13 Marsoni S, Bertotti A, Sartore-Bianchi A, Leone F, Lonardi S, Ciardiello F, et al: Dual anti-HER2 treatment of patients with HER2-positive metastatic colorectal cancer: the HERACLES trial (HER2 Amplification for ColorectaL Cancer Enhanced Stratification). J Clin Oncol 2013;31(suppl; abstr TPS3648).

14 Iqbal N, Iqbal N: Human epidermal growth factor receptor 2 (HER2) in cancers: overexpression and therapeutic implications. Mol Biol Int 2014;2014:852748.
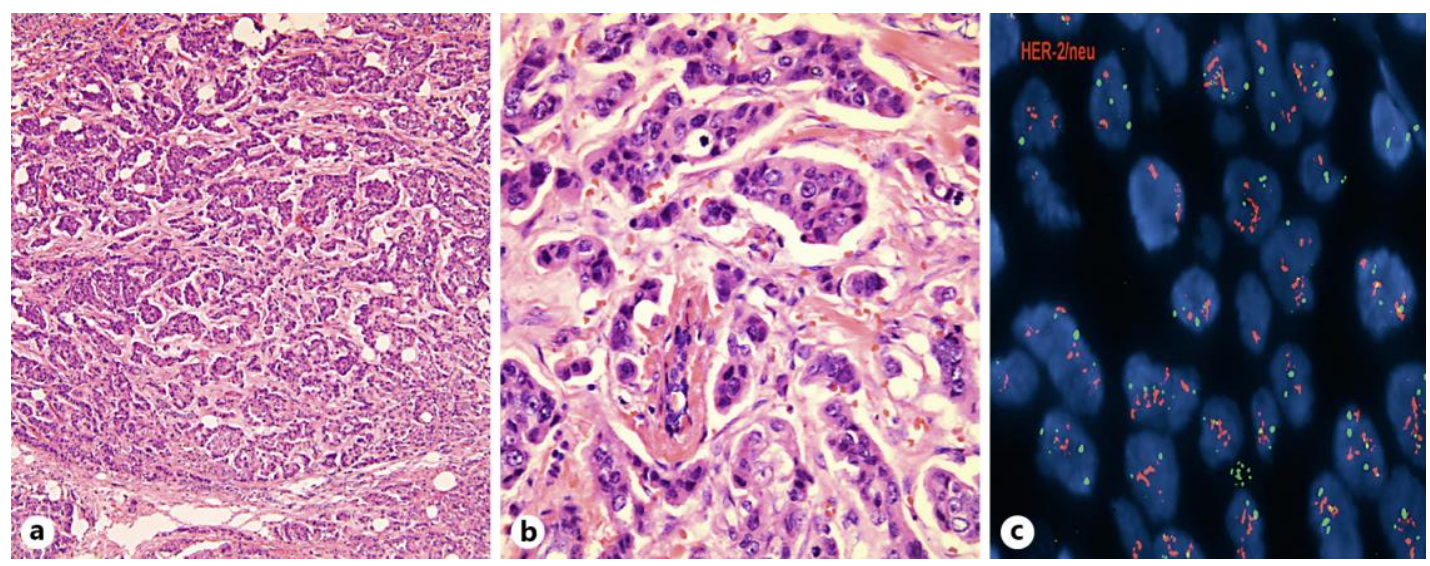

Fig. 1. Case 1, left parotid mass: HER-2 immunohistochemistry demonstrates overexpression at $\times 100$ (a) magnification and $\times 400$ (b) magnification. 
Shin et al.: Human Epidermal Growth Factor Receptor 2 (HER-2/neu)-Directed Therapy for Rare Metastatic Epithelial Tumors with HER-2 Amplification
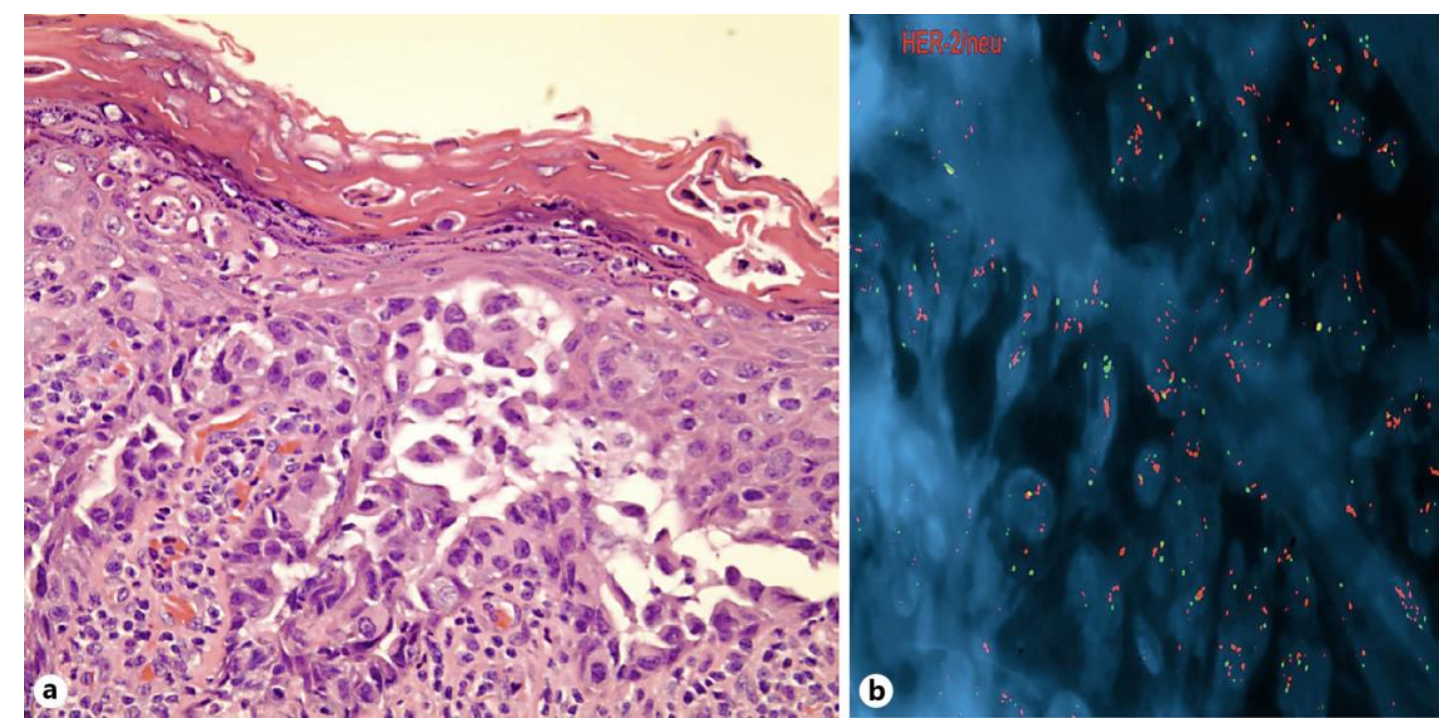

Fig. 2. Case 2, scrotal biopsy: a HER-2 immunohistochemistry demonstrates overexpression at $\times 400$ magnification; b fluorescence in situ hybridization analysis demonstrates HER-2 amplification. 